\title{
THE ISOMORPHISM QUESTION FOR DIVISION RINGS OF GROUP RINGS
}

\author{
D. R. FARKAS, A. H. SCHOFIELD, R. L. SNIDER AND J. T. STAFFORD
}

ABSTRACT. The division ring of fractions of the group ring of a torsion free finitely generated nilpotent group determines the group up to isomorphism.

1. Introduction. Very little seems to be known about division algebras infinite dimensional over their centers. There are few obvious candidates for invariants. As a consequence, it is generally a difficult problem to decide whether two given division algebras are isomorphic. The reader may recall the intricate dimension invented by Gel'fand and Kirillov to distinguish between the division rings of fractions of Weyl algebras with different indices [1].

In this paper we study a related class of division algebras. Assume that $k$ is a (commutative) field and that $G$ is a finitely generated torsion free nilpotent group. In this case $G$ can be fully ordered [6, Lemma 1.6, p. 587]. By using this ordering much the way one uses the degree function in a polynomial ring, one proves that the (algebraic) group algebra $k[G]$ has no zero divisors (cf. [6, p. 582]). By a well-known adaptation of the Hilbert Basis Theorem, $k[G]$ is a Noetherian ring [2]. Putting these observations and the Goldie Theorems together, we see that $k[G]$ has a classical division ring of fractions, which we denote $Q(k[G])$. The structure of these division rings is a mystery. Here we establish the following surprising result.

MAIN THEOREM. Suppose $G_{1}$ and $G_{2}$ are finitely generated torsion free nilpotent groups. If $Q\left(k\left[G_{1}\right]\right)$ and $Q\left(k\left[G_{2}\right]\right)$ are isomorphic division algebras over $k$, then $G_{1}$ and $G_{2}$ are isomorphic groups.

This proposition is unexpected in light of the Gel'fand-Kirillov structure theorem for the division ring of fractions of the enveloping algebra of a finite-dimensional nilpotent Lie algebra [1]. There one always obtains the ring of fractions of a Weyl algebra over a purely transcendental extension field of $k$; there are lots of coincidences.

The technique introduced in this paper is really a very old construction going back to Hilbert [3]. Utilizing the aforementioned ordering, one embeds $Q(k[G])$ in a far more tractable division algebra, which is equipped with something like a degree function mapping $Q(k[G])^{*}$ onto $G$. This and another similar function are used to provide an isomorphism between any two nilpotent groups $G$ and $H$ with $Q(k[G]) \simeq Q(k[H])$.

We end by noting that isomorphism results like the above theorem are going to hold in only very restricted circumstances. For example, let $H$ be a poly-(infinitecyclic) group. Then $H$ is right ordered [6, Lemma 1.6, p. 587]. Again $k[H]$ is a

Received by the editors December 7, 1981.

1980 Mathematics Subject Classification. Primary 16A39, 16A27, 20 C07.

Key words and phrases. Division ring, nilpotent group, group ring.

(C) 1982 American Mathematical Society 0002-9939/81/0000-0668/\$02.00 
Noetherian domain with a quotient division ring $Q(k[H])[6$, p. 591]. However this ring is no longer an invariant for $H$; we provide an example of poly-(infinite-cyclic), indeed abelian-by-finite, groups $G$ and $H$ such that $Q(k[G]) \simeq Q(k[H])$ but $G \not$ $H$.

2. The proof. We begin by briefly reviewing a construction due to Hilbert [3] as generalized and twisted by B. H. Neumann [5].

Let $F$ be a field and let $G$ be an ordered group. Suppose that $c: G \times G \rightarrow F^{*}$ is a normalized cocycle (i.e. $c(x, 1)=c(1, x)=1$ for all $x \in G)$. The set $H(G, F ; c)$ is the collection of all formal sums $\sum a_{x} \dot{x}$ where $a_{x} \in F, x \in G$, and $\left\{x \in G \mid a_{x} \neq\right.$ $0\}$ is well ordered under the ordering on $G$. The fundamental result (cf. $[6, \mathrm{p}$. $601])$ is that $H$ is a division ring under pointwise addition and multiplication which "linearly" extends $\left(a_{x} \dot{x}\right) \cdot\left(b_{y} \dot{y}\right)=a_{x} b_{y} c(x, y)(\dot{x} y)$.

We shall take advantage of the "lowest term" map $L: H(G, F ; c) \rightarrow F^{*} \cdot G$. It is defined so that $L\left(\sum a_{x} \dot{x}\right)=a_{y} y$ where $y$ is the smallest element of $G$ with a nonzero coefficient in the sum. It is easy to check that $L$ is a group homomorphism.

We next establish notation that will remain constant for the rest of the paper. $k$ is a (commutative) field. $G$ denotes a finitely generated torsion free nilpotent group. The field of fractions of $k[Z(G)]$ is denoted by $K$. It is well known that $G / Z(G)$ is also a finitely generated torsion free nilpotent group and so, by earlier remarks, it can be fully ordered. Pick coset representatives $\dot{x}$ in $G$ for $x \in G / Z(G)$ with $\dot{1}=1$ and let $f \in H^{2}(G / Z(G), Z(G))$ be defined by $\dot{x} \cdot \dot{y}=f(x, y)(\dot{x} y)$. The inclusion of $Z(G)$ in $K^{*}$ induces a homomorphism $i^{*}: H^{2}(G / Z(G), Z(G)) \rightarrow H^{2}\left(G / Z(G), K^{*}\right)$. Let $H$ be the division ring $H\left(G / Z(G), K ; i^{*}(f)\right)$. We write $\tilde{G}$ for the subgroup $K^{*} \cdot(G / Z(G))$ of $H^{*}$; and fix $L: H^{*} \rightarrow \tilde{G}$ as the lowest term homomorphism.

There are several straightforward properties of $H$ whose proofs are left as exercises. Of them, the most important observation is that the group algebra $k[G]$ has an obvious embedding in $H$. (Notice that $G$ is embedded in $\tilde{G}$.) By the universal property of fractions, the embedding of $k[G]$ extends to an embedding of $Q(k[G])$ in $H$.

Direct calculation shows that the centralizer of $G / Z(G)$ in $H$ is $K$. Consequently $K$ is the center of both $H$ and $Q(k[G])$ and, as such, has an invariant description. We next show that the group $\tilde{G}$ obtained from this construction is also an invariant for $G$.

PROPOSITION 1. Let $G_{1}$ and $G$ be finitely generated torsion free nilpotent groups with $Q\left(k\left[G_{1}\right]\right) \stackrel{\theta}{\simeq} Q(k[G])$. Let $L$ be the lowest term map defined by the ordering of $G / Z(G)$. Then $\left.L\right|_{\tilde{G}_{1}}$ is an isomorphism between $\tilde{G}_{1}$ and $\tilde{G}$.

Proof. Identify $Q\left(k\left[G_{1}\right]\right)=Q(k[G]) \subseteq H$ and observe that $\left.L\right|_{K^{*}}$ is the identity. Since $\tilde{G}_{1}$ is nilpotent with center $K^{*}$, this implies that $\left.L\right|_{\tilde{G}_{1}}$ is injective. Let $\left\{t_{i} \mid i \in I\right\}$ be a transversal for $K^{*}$ in $\tilde{G}_{1}$. As $L\left(K^{*}\right)=K^{*}$ and $L$ is injective on $\tilde{G}_{1}$, the $L\left(t_{i}\right)$ lie in different cosets of $K^{*}$ in $\tilde{G}$. This means that the $L\left(t_{i}\right)$ have differing lowest terms in the ordering of $G / Z(G)$. Let $\alpha \neq 0 \in k[G]$; say $\alpha=\sum \alpha_{i} t_{i}$ for some $\alpha_{i} \in K^{*}$. Then $L(\alpha)=L\left(\alpha_{j} t_{j}\right)$ where $t_{j}$ is the lowest term in the support of $\alpha$. Therefore $L(\alpha) \in L\left(\tilde{G}_{1}\right)$. If $d \neq 0 \in Q(k[G])$, then $d=\alpha^{-1} \beta$ for $\alpha, \beta \in k\left[G_{1}\right]$ and $L(d)=L(\alpha)^{-1} L(\beta) \in L\left(\tilde{G}_{1}\right)$. Since $L(\tilde{G})=\tilde{G}$, this implies that $L$ is onto. 
LEMMA 2. If $G$ is a finitely generated torsion free nilpotent group, then $\tilde{G}=$ $k^{*} \times G \times P$ where $P$ is a free abelian group.

Proof. $K^{*}=Q(k[Z(G)])$ and $k[Z(G)]$ is a unique factorization domain. Thus $K^{*}=k^{*} \times Z(G) \times P$ where $P$ is free on representatives for the prime elements of $k[Z(G)]$. Since $\tilde{G}=K^{*} \cdot G$, this decomposition extends to give $\tilde{G}=k^{*} \times G \times P$.

COROLlARY 3. Suppose that $G_{1}$ and $G_{2}$ are finitely generated torsion free nilpotent groups with $Q\left(k\left[G_{1}\right]\right) \simeq Q\left(k\left[G_{2}\right]\right)$. Then $G_{1} \times Z \simeq G_{2} \times Z$, where $Z$ is the infinite cyclic group.

PrOOF. By the proposition, there exists an isomorphism $L: \tilde{G}_{1} \rightarrow \tilde{G}_{2}$ and by Lemma 2, $\tilde{G}_{i}=k^{*} \times G_{i} \times P_{i}$. As $L\left(k^{*}\right)=k^{*}$, we may cancel $k^{*}$ to obtain an isomorphism $\varphi: G_{1} \times P_{1} \rightarrow G_{2} \times P_{2}$. Identify $G_{1} \times P_{1}$ with $G_{2} \times P_{2}$. Then $G_{1} \subseteq G_{2} \times F$ for a finitely generated subgroup $F$ of $P_{2}$. By the modular law $G_{2} \times F=G_{1} \times\left[\left(G_{2} \times F\right) \cap P_{1}\right]$ and so $G_{2} \times F \simeq G_{1} \times F_{1}$ for free abelian groups $F$ and $F_{1}$ of finite rank. The isomorphism $\varphi$ implies that $G_{2} / Z\left(G_{2}\right) \simeq G_{1} / Z\left(G_{1}\right)$. The rank of $Z\left(G_{i}\right)$ equals the transcendence degree of $K$ and thus $G_{1}$ and $G_{2}$ have the same Hirsch number; whence $F \simeq F_{1}$. Finally, by [4, Lemma 1], this implies that $G_{1} \times Z \simeq G_{2} \times Z$.

If $G_{1}$ is nilpotent of class two or if $G_{1} \simeq Z \times H$ for some group $H$, then $G_{1} \times Z \simeq G_{2} \times Z$ actually implies that $G_{1} \simeq G_{2}$ (see [4]). So Corollary 3 actually proves the Theorem in these two cases. In the general case the next lemma will be needed.

LEMMA 4. Let $G$ and $H$ be finitely generated torsion free groups such that $G \times$ $P_{1} \stackrel{\theta}{\simeq} H \times P_{2}$ for abelian groups $P_{i}$. Let $\varphi: H \times P_{2} \rightarrow H$ be a homomorphism with $\left.\varphi\right|_{H}=\mathrm{Id}$ and set $\psi=\left.\varphi \theta\right|_{G}$. Then $\operatorname{Ker} \psi$ is abelian and $G \simeq(\operatorname{Ker} \psi) \times \psi(G)$.

Proof. As $\theta\left(G^{\prime}\right) \subseteq H$, clearly $\left.\psi\right|_{G^{\prime}}$ is one-to-one. Write $K=\operatorname{Ker} \psi$. Now $[K, G] \subseteq G^{\prime} \cap K=1$; whence $K$ is central and $K \cap G^{\prime}=1$. In order to prove that $K$ is a direct summand, it suffices to show that $K G^{\prime} / G^{\prime}$ is a pure subgroup of $G / G^{\prime}$. So suppose that $x^{p} \in K G^{\prime}$; say $x^{p}=k t$ for $k \in K$ and $t \in G^{\prime}$. Write $\theta(k)=(g, f)$ and $\theta(t)=h$ for some $g, h \in H$ and $f \in P_{2}$. Then $\theta(x)^{p}=\theta(k t)=(g h, f)$, and so $f=f_{1}^{p}$ for some $f_{1} \in P_{2}$. Now $1=\psi(k)=\varphi\left(g, f_{1}^{p}\right)=g \varphi\left(f_{1}^{p}\right)$ and so $g=g_{1}^{p}$. Thus $\theta(k)=\left(g_{1}, f_{1}\right)^{p}$ and $k=z^{p}$ for some $z \in G$. But $1=\psi(k)=\psi(z)^{p}$ which, as $H$ is torsion free, implies that $z \in K$ as required.

Proof of the MAIN Theorem. By Lemma $3, G_{1} \times Z \simeq G_{2} \times Z$. So, if $G_{1}$ has an infinite-cyclic factor, then [4, Lemma 1] implies that $G_{1} \simeq G_{2}$.

Thus we may assume that $G_{1}$ has no infinite-cyclic factors. In this case we will consider two distinct Hilbert-Neumann constructions. Pick an ordering on $G_{2}$ that induces an ordering on $G_{2} / Z\left(G_{2}\right)$. (Such orderings exist by the proof of $[6$, Lemma 1.5 , p. 587].) First let $T$ be the Hilbert-Neumann division $\operatorname{ring} T=H\left(G_{2}, k ; c\right)$ where $c(x, y)=1$. Then there exists a homomorphism $V: T^{*} \rightarrow G_{2}$ defined by $V\left(\sum f_{i} y_{i}\right)=\min \left\{y_{i} \mid f_{i} \neq 0\right\}$ for $y_{i} \in G_{2}$ and $f_{i} \in k$. We will show that $V$ provides the required isomorphism between $G_{1}$ and $G_{2}$, but to prove this we need to consider a second Hilbert-Neumann division ring. Let $K=Q\left(k\left[Z\left(G_{2}\right)\right]\right) \subseteq T$. Then the induced ordering on $G_{2} / Z\left(G_{2}\right)$ provides a division ring $H=H\left(G_{2}, K ; i^{*}(f)\right)$ as in the beginning of this section. Let $\left\{x_{i} \mid i \in I\right\}$ be a transversal for $Z\left(G_{2}\right)$ in $G_{2}$, and identify $H$ with the subring of $T$ consisting of all power series $\left\{\sum f_{i} x_{i} \mid f_{i} \in K\right.$ and 
$\left\{x_{i} \mid f_{i} \neq 0\right\}$ is well ordered $\}$. Let $L: H^{*} \rightarrow G_{2}$ be the homomorphism given by the induced ordering on $G_{2} / Z\left(G_{2}\right)$ and identify $Q\left(k\left[G_{1}\right]\right)$ with $Q\left(k\left[G_{2}\right]\right)$. Then by Proposition 1 and Lemma 2, $L$ induces a homomorphism $L: G_{1} \times A \rightarrow G_{2} \times B$ for abelian groups $A$ and $B$. Now $\left.V\right|_{G_{2}}=$ Id. Since $G_{1}$ has no cyclic factors, Lemma 4 therefore implies that $\left.V \circ L\right|_{G_{1}}$ is injective. Further, as the ordering on $G_{2} / Z\left(G_{2}\right)$ was the one induced from that on $G_{2}, V \circ L=\left.V\right|_{H}$. So $\left.V\right|_{G_{1}}$ is injective. To show that $\left.V\right|_{G_{1}}$ is surjective, just repeat the last half of the proof of Proposition 1.

We now provide an example of torsion free polycyclic groups $G_{1} \not G_{2}$ for which $Q\left(k\left[G_{1}\right]\right) \simeq Q\left(k\left[G_{2}\right]\right)$. As the examples are even abelian-by-finite and poly(infinite-cyclic), this shows that the Main Theorem is close to being the best possible result.

EXAMPLE. Let $G=\left\langle x, y, z \mid x \in Z(G), z y z^{-1}=y^{-1}\right\rangle$ and $H=\langle a, b, c| a b=$

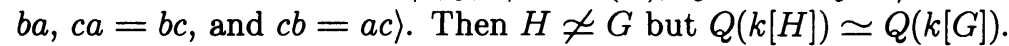

Proof. As $G^{\prime}=\left\langle y^{2}\right\rangle, G / G^{\prime}$ is not torsion free. However, $H^{\prime}=\left\langle a b^{-1}\right\rangle$ and so $H / H^{\prime}$ is torsion free. Thus $G \not t H$.

Let $x_{1}=a+b, y_{1}=a b^{-1}$, and $z_{1}=c$ belong to $k[H]$. Let $R$ be the subalgebra of $Q(k[H])$ generated by $x_{1}^{ \pm 1}, y_{1}^{ \pm 1}$, and $z_{1}^{ \pm 1}$. Clearly $R \simeq k[G]$. But $a=(a+b)\left(a b^{-1}\right)\left(a b^{-1}+1\right)^{-1}$, whence $a$ and $b=c a c^{-1}$ both belong to $Q(R)$. Thus $Q(k[H]) \simeq Q(R) \simeq Q(k[G])$.

Observe that in the above example, $G$ has an infinite cyclic factor whereas $H$ does not. Thus by [4, Lemma 1], $G$ and $H$ are not even stably isomorphic; i.e. $G \times F \not L H \times F$ for any free abelian group $F$ of finite rank. We remark, however, that one can at least recover the Hirsch number of a polycyclic-by-finite group $G$ from its division ring of fractions $Q(k[G])$ (see [7]).

\section{REFERENCES}

1. I. M. Gel'fand and A. A. Kirillov, Sur les corps liés aux algèbres enveloppantes des algèbres de Lie, Inst. Hautes Études Sci. Publ. Math. 31 (1966), 5-19.

2. P. Hall, Finiteness conditions for soluble groups, Proc. London Math. Soc. (3) 4 (1954), 419-436.

3. D. Hilbert, Grundlagen der Geometrie, 7th ed., Leipzig, 1930.

4. R. Hirshon, Some cancellation theorems with applications to nilpotent groups, J. Austral. Math. Soc. Ser. A 23 (1977), 147-165.

5. B. H. Neumann, On ordered division rings, Trans. Amer. Math. Soc. 66 (1949), 202-252.

6. D. S. Passman, The algebraic structure of group rings, Wiley-Interscience, New York, 1977.

7. J. T. Stafford, Dimensions of division rings (to appear).

Department of Mathematics, Virginia Polytechnic institute and State UNIVERSITY, BLACKSBURG, VIRGINIA 24061 (Current address of D. R. Farkas)

Department of MATHEMATICS, BedFord College, London NW1 4NS, ENGLAND (Current address of A. H. Schofield)

Department of Mathematics, CAMBridge University, Cambridge, ENGLAND (Current address of R. L. Snider)

Department of mathematics, Gonville and Caius College, Cambridge CB 2 1TA, ENGLAND

Current address (J. T. Stafford): Department of Mathematics, Leeds University, Leeds, England 